\title{
Analytical solution of advection-diffusion equation in heterogeneous infinite medium using Green's function method
}

\author{
AbHisheK SAnskrityayn* and NAVEen Kumar \\ Department of Mathematics, Institute of Science, Banaras Hindu University, Varanasi 221 005, India. \\ ${ }^{*}$ Corresponding author.e-mail: abhi.bhu2008@gmail.com
}

Some analytical solutions of one-dimensional advection-diffusion equation (ADE) with variable dispersion coefficient and velocity are obtained using Green's function method (GFM). The variability attributes to the heterogeneity of hydro-geological media like river bed or aquifer in more general ways than that in the previous works. Dispersion coefficient is considered temporally dependent, while velocity is considered spatially and temporally dependent. The spatial dependence is considered to be linear and temporal dependence is considered to be of linear, exponential and asymptotic. The spatio-temporal dependence of velocity is considered in three ways. Results of previous works are also derived validating the results of the present work. To use GFM, a moving coordinate transformation is developed through which this ADE is reduced into a form, whose analytical solution is already known. Analytical solutions are obtained for the pollutant's mass dispersion from an instantaneous point source as well as from a continuous point source in a heterogeneous medium. The effect of such dependence on the mass transport is explained through the illustrations of the analytical solutions.

\section{Introduction}

The pollutants solute transport due to advection and diffusion through a medium is described by a partial differential equation of parabolic type, derived on the principle of conservation of mass, known as advection-diffusion equation (ADE). In one dimension, it has two coefficients: diffusion coefficient and velocity. ADE is efficiently used to describe solute transport through a medium away from its source over long distances in open media like rivers as well as in porous media like aquifers. In the latter case, the velocity satisfies the Darcy law. But solute transport through heterogeneous medium cannot be described properly by ADE with constant coefficients (Simpson 1978;
Matheron and de Marsily 1980; Pickens and Grisak 1981a). Scale-dependent dispersion in heterogeneous hydrological systems was modelled by timedependent dispersion coefficient of linear, exponential, parabolic and asymptotic forms (Pickens and Grisak 1981b). The model was handled using finite element (linear triangular) method. This theory has been strengthened by field experiments (Anderson 1979; Gelhar et al. 1985) and by theoretical developments (Sposito et al. 1986; Dagan 1987). Theoretical deterministic analysis (Güven et al. 1984) has also established the temporal dependence of the dispersion coefficient in a stratified aquifer.

A generalized analytical transient, one-, two-, and/or three-dimensional (AT 123D) computer

Keywords. Advection; diffusion; heterogeneity; non-degenerate form; Green's function method. 
code was developed for estimating the transport of wastes in the groundwater aquifer system (Yeh 1981) which contains 450 options of types of wastes, source configurations, source of release, boundary conditions, and aquifer dimensions and dimensions of the Cartesian frame of references. It is recognized as a pioneer work using a Green function method to develop solute transport model, though the coefficients of the $\mathrm{ADE}$ were assumed constant. The analytical solution of one-dimensional ADE with temporally dependent dispersion coefficient and uniform velocity in an infinite heterogeneity medium was obtained by Basha and El-Habel (1993) using Green's function method for the instantaneous and continuous point sources. Later this work was extended in two dimensions (Aral and Liao 1996) and the analytical solutions were obtained using the same method. In both the works, a transformation introducing new time-domain has been used to get the ADE with constant coefficients. The objective of the study (Leij and Van Genuchten 2000) was to formulate Green's function for the equilibrium and non-equilibrium ADE, and to use Green's function method to derive analytical solution for solute movement. The work (Park and Zhan 2001) provides analytical solution of contaminant transport from one-, two-, and threedimensional finite sources in a finite-thickness aquifer, using Green's function. The analytical solution was obtained to predict the contaminant concentration along non-uniform groundwater flow in semi-infinite medium (Singh et al. 2008). The analytical solutions have been presented to onedimensional advection-diffusion equation with spatially dependent dispersion along non-uniform flow through inhomogeneous medium in which solute dispersion is assumed proportional to the square of velocity (Kumar et al. 2009). A new analytical solution was presented for the cross-sectional integrated one-dimensional ADE with spatially dependent coefficients (Zamani and Bombardelli 2012). A series of one- and multidimensional solutions of ADE with or without accounting for zero-order production and first order decay was presented (Van Genuchten et al. 2013), which has been useful for simplified analysis of contaminant transport in surface water, and for mathematical verification of more comprehensive numerical transport models.

To account the mixing caused by velocity fluctuations, dispersion coefficient was considered as a function of both space and time variables in the porous media in a theoretical and experimental work by Sternberg et al. (1996). On the basis of this paper, the dispersion parameter has been expressed in both the independent variables but in degenerate form (Su et al. 2005; Yadav et al. 2011). To accommodate the effects of winds upon solute transport in open tidal fjords channel, the velocity has been considered as function of depth variable and sinusoidally varying time variable (Wang et al. 1977). In the present work, the heterogeneity of the medium is delineated in a more general way by considering velocity dependence on both the independent variables and dispersion parameter dependence on the time variable. Analytical solutions of one-dimensional ADE with these two coefficients are obtained in an infinite domain subjected to the instantaneous and continuous injected sources using Green's function method. To use this method, a moving coordinate transformation is developed. It helps to get the solution of the ADE in the general form from which solutions are obtained for different particular cases including those of earlier works. The next section comprises of the mathematical formulation of the problem and its analytical solutions for instantaneous and continuous sources, and a subsection of particular cases. It is followed by a section on the discussion of the results based on their illustrations through figures. Lastly, concluding remarks and a list of references are given.

\section{Mathematical formulation and analytical solution}

Let us consider linear ADE in one dimension in general form as:

$$
\frac{\partial c}{\partial t}=\frac{\partial}{\partial x}\left[D_{0} \psi(t) \frac{\partial c}{\partial x}-u_{0} f(x, t) c\right]+q(x, t),
$$

where $c$ is the solute concentration at position $x$ and at time $t, D_{0}$ and $u_{0}$ are uniform dispersion coefficient and uniform velocity, respectively, in a homogeneous medium, $q(x, t)$ is the injection term for the solute mass in the infinite domain. As discussed in the previous section, the medium is considered heterogeneous, so the two coefficients of the $\mathrm{ADE}$, the dispersion coefficient $D(x, t)$ and velocity $u(x, t)$ are considered temporally dependent and spatio-temporally dependent, and are written as $D_{0} \psi(t)$ and $u_{0} f(x, t)$, respectively, in equation (1). This equation may be written in expanded form as:

$$
\frac{\partial c}{\partial t}=D_{0} \psi(t) \frac{\partial^{2} c}{\partial x^{2}}-u_{0} f(x, t) \frac{\partial c}{\partial x}-u_{0} \frac{\partial f}{\partial x} c+q(x, t) .
$$

First objective is to reduce the above equation into a form, whose analytical solution is known and in that process, the expression for $f(x, t)$ is obtained. Let equation (2) be transformed into an equation

$$
\begin{aligned}
\frac{\partial C}{\partial t^{\prime}}= & D_{0} \psi_{1}\left(t^{\prime}\right) \frac{\partial^{2} C}{\partial X^{2}}-u_{0} \frac{\partial C}{\partial X} \\
& -\alpha g\left(t^{\prime}\right) C+q_{1}\left(X, t^{\prime}\right),
\end{aligned}
$$


in a new domain $\left(X, t^{\prime}\right)$ through coordinate transformation equations

$$
X=X(x, t), t^{\prime}=t .
$$

It may be noted that while doing so, the function of the temporally dependent coefficient of secondorder space derivative also changes. The analytical solution of a similar ADE is given by Basha and El-Habel (1993). In equation (3), the decay term is also assumed varying with time for generality, where $\alpha$ is a parameter, which has dimension of inverse of time variable. Equation (3) is dimensionally consistent as $\psi_{1}\left(t^{\prime}\right)$ and $g\left(t^{\prime}\right)$ are dimensionless expressions. It is evident in the later part of this paper. Using the transformation equation (4), equation (2) may be written as equation (5) (now onwards in place of $t^{\prime}$ we are using only $t$ for the sake of convenience)

$$
\begin{aligned}
\frac{\partial C}{\partial t}= & D_{0} \psi(t)\left(\frac{\partial X}{\partial x}\right)^{2} \frac{\partial^{2} C}{\partial X^{2}} \\
& +\left[D_{0} \psi(t) \frac{\partial^{2} X}{\partial x^{2}}-u_{0} f(x, t) \frac{\partial X}{\partial x}-\frac{\partial X}{\partial t}\right] \frac{\partial C}{\partial X} \\
& -u_{0} \frac{\partial f(x, t)}{\partial x} C+q_{1}(X, t)
\end{aligned}
$$

Equation (5) will become equation (3) if equations $(6 \mathrm{a}, \mathrm{b}$ and $\mathrm{c})$ are followed

$$
\begin{gathered}
\psi(t)\left(\frac{\partial X}{\partial x}\right)^{2}=\psi_{1}(t), \\
D_{0} \psi(t) \frac{\partial^{2} X}{\partial x^{2}}-u_{0} f(x, t) \frac{\partial X}{\partial x}-\frac{\partial X}{\partial t}=-u_{0},
\end{gathered}
$$

and

$$
u_{0} \frac{\partial f(x, t)}{\partial x}=\alpha g(t)
$$

Solving (6c), $f(x, t)$ may be obtained; hence, the expression for velocity may be written as:

$$
u(x, t)=u_{0} f(x, t)=\alpha x g(t)+u_{0} \phi(t) .
$$

Integrating equation (6a) with respect to $x$, we have

$$
X=\int \sqrt{\frac{\psi_{1}(t)}{\psi(t)}} d x+\phi_{1}(t) .
$$

Using equations (6a, 7 and 8) into equation (6b), we get:

$$
\begin{aligned}
& u_{0}-\left\{\alpha x g(t)+u_{0} \phi(t)\right\} \sqrt{\frac{\psi_{1}(t)}{\psi(t)}} \\
& =\frac{\partial}{\partial t}\left(\int \sqrt{\frac{\psi_{1}(t)}{\psi(t)}} d x+\phi_{1}(t)\right) .
\end{aligned}
$$

Simplifying equation (9), we get:

$$
\begin{aligned}
& \left(\frac{d \phi_{1}}{d t}-u_{0}+u_{0} \phi(t) \sqrt{\frac{\psi_{1}(t)}{\psi(t)}}\right) x^{0} \\
& +\left(\frac{d}{d t} \sqrt{\frac{\psi_{1}(t)}{\psi(t)}}+\alpha g(t) \sqrt{\frac{\psi_{1}(t)}{\psi(t)}}\right) x=0 .
\end{aligned}
$$

Equating the coefficients of $x^{0}$ and $x^{1}$ from both sides in equation (10) we have:

$$
\frac{d}{d t} \sqrt{\frac{\psi_{1}(t)}{\psi(t)}}=-\alpha g(t) \sqrt{\frac{\psi_{1}(t)}{\psi(t)}},
$$

and

$$
\frac{d \phi_{1}}{d t}=u_{0}-u_{0} \phi(t) \sqrt{\frac{\psi_{1}(t)}{\psi(t)}} .
$$

From equation (11a) we have:

$$
\psi_{1}(t)=\frac{\psi(t)}{\beta^{2}(t)},
$$

where

$$
\beta(t)=\exp \left(\alpha \int_{0}^{t} g(v) d v\right),
$$

is a dimensionless expression. Further using $\psi_{1}(t)$ in equation $(11 \mathrm{~b})$ we get:

$$
\frac{d \phi_{1}}{d t}=u_{0}-u_{0} \frac{\phi(t)}{\beta(t)} .
$$

Using equation (7) for $f(x, t)$, equation (1) may be considered as:

$$
\begin{aligned}
\frac{\partial c}{\partial t}= & \frac{\partial}{\partial x}\left[D_{0} \psi(t) \frac{\partial c}{\partial x}-\left\{\alpha x g(t)+u_{0} \phi(t)\right\} c\right] \\
& +q(x, t) ; \quad-\infty<x<\infty, t>0 .
\end{aligned}
$$

Equation (15) may be reduced into the form

$$
\frac{\partial C}{\partial t}=D_{0} \frac{\psi(t)}{\beta^{2}(t)} \frac{\partial^{2} C}{\partial X^{2}}-u_{0} \frac{\partial C}{\partial X}-\alpha g(t) C+q_{1}(X, t),
$$

by using the transformation

$$
X=\frac{x}{\beta(t)}+u_{0} t-u_{0} \int_{0}^{t} \frac{\phi(v)}{\beta(v)} d v
$$

where $\beta(t)$ is defined in equation (13). The variable $X$ is also a space variable. To complete the formulation, an initial condition is assumed as:

$$
C(X, 0)=C_{i} \omega(X) ; \quad-\infty<X<\infty .
$$


The transformation equation

$$
\eta=X-u_{0} t
$$

is used to eliminate the convective term from equation (16). Another transformation equation

$$
K(\eta, t)=C(\eta, t) \beta(t)
$$

is used to eliminate the first-order decay term, and lastly, the transformation equation (Crank 1975)

$$
T=\int_{v=0}^{t} \frac{\psi(v)}{\beta^{2}(v)} d v
$$

is used. As a result, equation (16) may be obtained in the form

$$
\frac{\partial K}{\partial T}=D_{0} \frac{\partial^{2} K}{\partial \eta^{2}}+\frac{Q(\eta, T)}{\psi(t)} \beta^{3}(t),
$$

where $T$ is another time variable. The initial condition becomes

$$
K(\eta, T=0)=C_{i} \omega(\eta) .
$$

Analytical solution of the problem described by equations (22 and 23) may be obtained by Green's method (Haberman 1987) as:

$$
\begin{aligned}
K(\eta, T)= & \int_{0}^{T} \int_{-\infty}^{\infty} \frac{\beta^{3}(\zeta)}{\sqrt{4 \pi D_{0}(T-\zeta)}} \frac{Q(\chi, \zeta)}{\psi(\zeta)} \\
& \times \exp \left(-\frac{(\eta-\chi)^{2}}{4 D_{0}(T-\zeta)}\right) d \chi d \zeta \\
& +C_{i} \int_{-\infty}^{\infty} \frac{\omega(\chi)}{\sqrt{4 \pi D_{0} T}} \exp \left(-\frac{(\eta-\chi)^{2}}{4 D_{0} T}\right) d \chi
\end{aligned}
$$

Using the transformations used earlier, the desired solution may be obtained as:

$$
\begin{aligned}
c(x, t)= & \int_{0}^{t} \int_{-\infty}^{\infty} \frac{Q\left(\chi, t_{0}\right)}{\sqrt{4 \pi D_{0}(T-\zeta)}} \frac{\beta\left(t_{0}\right)}{\beta(t)} \exp \left[-\frac{1}{4 D_{0}(T-\zeta)}\right. \\
& \left.\times\left(\frac{x}{\beta(t)}-u_{0} \int_{0}^{t} \frac{\phi(v)}{\beta(v)} d v-\chi\right)^{2}\right] d \chi d t_{0} \\
& +\frac{C_{i}}{\beta(t)} \int_{-\infty}^{\infty} \frac{\omega(\chi)}{\sqrt{4 \pi D_{0} T}} \\
& \times \exp \left[-\frac{1}{4 D_{0} T}\left(\frac{x}{\beta(t)}-u_{0} \int_{0}^{t} \frac{\phi(v)}{\beta(v)} d v-\chi\right)^{2}\right] d \chi
\end{aligned}
$$

where using equation (21), the new time variable, $T$ may be expressed in terms of old time variable, $t$ for different expressions of $\psi(t)$. The above solution may be expressed in more concise form by considering $\xi=\chi \beta\left(t_{0}\right)$, so $d \xi=\beta\left(t_{0}\right) d \chi$ may be used in the first integral. Finally, the analytical solution in equation (25) may be written as:

$$
\begin{aligned}
c(x, t) & =\frac{1}{\beta(t)} \int_{0}^{t} \int_{-\infty}^{\infty} \frac{Q_{1}\left(\xi, t_{0}\right)}{\sqrt{4 \pi D_{0}(T-\zeta)}} \exp \left[-\frac{1}{4 D_{0}(T-\zeta)}\right. \\
& \left.\times\left(\frac{x}{\beta(t)}-u_{0} \int_{0}^{t} \frac{\phi(v)}{\beta(v)} d v-\frac{\xi}{\beta\left(t_{0}\right)}\right)^{2}\right] d \xi d t_{0} \\
& +\frac{C_{i}}{\beta(t)} \int_{-\infty}^{\infty} \frac{\omega(\chi)}{\sqrt{4 \pi D_{0} T}} \\
& \times \exp \left[-\frac{1}{4 D_{0} T}\left(\frac{x}{\beta(t)}-u_{0} \int_{0}^{t} \frac{\phi(v)}{\beta(v)} d v-\chi\right)^{2}\right] d \chi
\end{aligned}
$$

where

$$
\begin{aligned}
& T=\int_{0}^{t} \frac{\psi(v)}{\beta^{2}(v)} d v, \quad \zeta=\int_{0}^{t_{0}} \frac{\psi(v)}{\beta^{2}(v)} d v \\
& Q_{1}\left(\xi, t_{0}\right)=q\left[\xi+u_{0} \beta\left(t_{0}\right) \int_{0}^{t_{0}} \frac{\phi(v)}{\beta(v)} d v, t_{0}\right] .
\end{aligned}
$$

The solutions describing the concentration distribution pattern from an instantaneous point injection and a continuous source of solute mass are obtained below.

\subsection{Instantaneous point injection}

Instantaneous non-dimensional injection of a solute mass is given by

$$
q(x, t)=M \delta(x) \delta(t),
$$

where $M$ is the injected mass, and $\delta(x)$ is the Dirac delta function. Further, we assume that the infinite domain is initially pollutant free, i.e.,

$$
c(x, 0)=0 .
$$

Using the property of Dirac delta function

$$
f(x)=\int_{-\infty}^{\infty} f\left(x_{0}\right) \delta\left(x_{0}-x\right) d x_{0},
$$


and

$$
f(t)=\int_{0}^{t} f\left(t_{0}\right) \delta\left(t_{0}-t\right) d t_{0},
$$

solution in equation (26) will provide for the solute transport from the instantaneous source as:

$$
\begin{aligned}
c(x, t)= & \frac{M}{\beta(t)} \frac{1}{\sqrt{4 \pi D_{0} T}} \\
& \times \exp \left[-\frac{1}{4 D_{0} T}\left(\frac{x}{\beta(t)}-u_{0} \int_{0}^{t} \frac{\phi(v)}{\beta(v)} d v\right)^{2}\right] .
\end{aligned}
$$

\subsection{Continuous injection}

Let it be defined as:

$$
q(x, t)=C_{0} u_{0} f(0, t) \delta(x), \quad t>0 .
$$

The solution for the solute transport from continuous source may be obtained from equation (26) as:

$$
\begin{aligned}
c(x, t) & =\frac{C_{0} u_{0}}{\beta(t)} \int_{0}^{t} \frac{\phi\left(t_{0}\right)}{\sqrt{4 \pi D_{0}(T-\zeta)}} \\
& \times \exp \left[-\frac{1}{4 D_{0}(T-\zeta)}\left(\frac{x}{\beta(t)}-u_{0} \int_{t_{0}}^{t} \frac{\phi(v)}{\beta(v)} d v\right)^{2}\right] d t_{0} .
\end{aligned}
$$

\subsection{Particular cases}

\subsubsection{Spatially dependent velocity and constant coefficient of decay term}

Let $g(t)=\phi(t)=1$. So, from equation (7), velocity has the expression $u(x, t)=u_{0}+\alpha x$, and from equation (13), $\beta(t)=\exp (\alpha t)$. The respective solutions for instantaneous injection and continuous injection may be obtained from equations (30 and 32) as:

$$
\begin{aligned}
c(x, t)= & M \frac{\exp (-\alpha t)}{\sqrt{4 \pi D_{0} T}} \exp \left[-\frac{1}{4 D_{0} T}(x \exp (-\alpha t)\right. \\
& \left.\left.+\frac{u_{0}}{\alpha}\{\exp (-\alpha t)-1\}\right)^{2}\right],
\end{aligned}
$$

and

$$
\begin{aligned}
c(x, t)= & C_{0} u_{0} \int_{0}^{t} \frac{\exp (-\alpha t)}{\sqrt{4 \pi D_{0}(T-\zeta)}} \exp \left[-\frac{1}{4 D_{0}(T-\zeta)}\right. \\
& \times\left(x \exp (-\alpha t)+\frac{u_{0}}{\alpha}\{\exp (-\alpha t)\right. \\
& \left.\left.\left.-\exp \left(-\alpha t_{0}\right)\right\}\right)^{2}\right] d t_{0} .
\end{aligned}
$$

Further, following four cases of temporally dependent dispersion coefficient are considered. In each case, using equation (21), respective expression for the new time variable $T$ may be obtained in both the above solutions.

(i) Constant dispersion coefficient:

Let $\psi(t)=1$, so $D(t)=D_{0}$. Using equation (21) we have:

$$
T=\frac{1}{2 \alpha}[1-\exp (-2 \alpha t)] .
$$

As $\alpha \rightarrow 0$, both dispersion coefficient and velocity become constant. Then, equation (33) becomes the solution of Yeh (1981), Haberman (1987) and Basha and El-Habel (1993) in case of instantaneous point source, and equation (34) becomes that of Yeh (1981), Beck et al. (1992) and Yeh and Yeh (2007) for continuous point source.

(ii) Linear dispersion coefficient:

Let $\psi(t)=t / k$, so $D(t)=D_{0}(t / k)$, and

$$
T=\frac{1}{4 k \alpha^{2}}\{1-\exp (-2 \alpha t)\}-\frac{t}{2 k \alpha} \exp (-2 \alpha t) \text {. }
$$

(iii) Exponential dispersion coefficient:

Let $\psi(t)=1-\exp (-t / k)$, so $D(t)=$ $D_{0}\{1-\exp (-t / k)\}$ and

$$
\begin{aligned}
T= & \frac{1}{2 \alpha}\{1-\exp (-2 \alpha t)\}+\frac{k}{2 \alpha k+1} \\
& \times\left\{\exp \left(-\frac{(2 \alpha k+1) t}{k}\right)-1\right\} .
\end{aligned}
$$

(iv) Asymptotic dispersion coefficient:

$$
\text { Let } \psi(t)=\frac{t}{t+k} \text {, so } D(t)=D_{0}(t /(t+k))
$$
and

$$
T=\int_{0}^{t} \frac{v}{v+k} e^{-2 \alpha v} d v .
$$

As $\alpha \rightarrow 0$, then solution given by equations (33 and 34) become the respective solutions for instantaneous source and continuous source given by Basha and El-Habel (1993). The parameter $k=0$ in above form leads to constant diffusion coefficient discussed in case (i). The integral (38) may not be evaluated directly, hence numerical integration is carried out using Simpson's rule.

\subsubsection{Spatially and temporally dependent velocity and constant coefficient of decay term}

To discuss this case, dispersion coefficient is considered asymptotic that is $D(t)=D_{0}(t /(t+k))$, so the new time variable $T$ has the expression given 
in equation (38). Also, let us consider $g(t)=1$, so from equation (7) spatially and temporally dependent velocity has the expression $u(x, t)=\alpha x+$ $u_{0} \phi(t)$. Three expressions of $\phi(t)$ are considered. For each one, the solutions in both the cases of injected mass are written.

(i) $\phi(t)=1-\exp (-m t)$, where $m$ is a temporaldependent parameter of dimension inverse of time variable. The two solutions (30) and (32) become

$$
\begin{aligned}
c(x, t)= & M \frac{\exp (-\alpha t)}{\sqrt{4 \pi D_{0} T}} \exp \left[-\frac{1}{4 D_{0} T}(x \exp (-\alpha t)\right. \\
& +\frac{u_{0}}{\alpha}\{\exp (-\alpha t)-1\}+\frac{u_{0}}{m+\alpha} \\
& \left.\times\{1-\exp (-m t-\alpha t)\})^{2}\right],
\end{aligned}
$$

and

$$
\begin{aligned}
c(x, t)= & C_{0} u_{0} \int_{0}^{t} \frac{\phi\left(t_{0}\right) \exp (-\alpha t)}{\sqrt{4 \pi D_{0}(T-\zeta)}} \\
& \times \exp \left[-\frac{1}{4 D_{0}(T-\zeta)}(x \exp (-\alpha t)\right. \\
& +\frac{u_{0}}{\alpha}\left\{\exp (-\alpha t)-\exp \left(-\alpha t_{0}\right)\right\} \\
& +\frac{u_{0}}{\alpha+m}\left\{\exp \left(-\alpha t_{0}-m t_{0}\right)\right. \\
& \left.-\exp (-\alpha t-m t)\})^{2}\right] d t_{0},
\end{aligned}
$$

respectively.

(ii) $\phi(t)=m t$. Using equations (30 and 32), we obtain the solutions for instantaneous and continuous injection sources as:

$$
\begin{aligned}
c(x, t) & =M \frac{\exp (-\alpha t)}{\sqrt{4 \pi D_{0} T}} \\
& \times \exp \left[-\frac{1}{4 D_{0} T}\left(x \exp (-\alpha t)+\frac{m t u_{0}}{\alpha}\right.\right. \\
& \left.\left.\times \exp (-\alpha t)+\frac{m u_{0}}{\alpha^{2}}\{\exp (-\alpha t)-1\}\right)^{2}\right],
\end{aligned}
$$

and

$$
\begin{aligned}
c(x, t) & =C_{0} u_{0} \int_{0}^{t} \frac{\phi\left(t_{0}\right) \exp (-\alpha t)}{\sqrt{4 \pi D_{0}(T-\zeta)}} \\
& \times \exp \left[-\frac{1}{4 D_{0}(T-\zeta)}(x \exp (-\alpha t)\right. \\
& -\frac{m u_{0}}{\alpha}\left\{t_{0} \exp \left(-\alpha t_{0}\right)-t \exp (-\alpha t)\right\} \\
& \left.\left.+\frac{m u_{0}}{\alpha^{2}}\left\{\exp (-\alpha t)-\exp \left(-\alpha t_{0}\right)\right\}\right)^{2}\right] d t_{0},
\end{aligned}
$$

respectively. (iii) $\phi(t)=\exp (m t)$. The two solutions (30) and (32) become

$$
\begin{aligned}
c(x, t)= & M \frac{\exp (-\alpha t)}{\sqrt{4 \pi D_{0} T}} \\
& \times \exp \left[-\frac{1}{4 D_{0} T}\left(x \exp (-\alpha t)+\frac{u_{0}}{\alpha-m}\right.\right. \\
& \left.\times\{1-\exp (-\alpha t+m t)\})^{2}\right],
\end{aligned}
$$

and

$$
\begin{aligned}
c(x, t)= & C_{0} u_{0} \int_{0}^{t} \frac{\phi\left(t_{0}\right) \exp (-\alpha t)}{\sqrt{4 \pi D_{0}(T-\zeta)}} \\
& \times \exp \left[-\frac{1}{4 D_{0}(T-\zeta)}(x \exp (-\alpha t)\right. \\
& +\frac{u_{0}}{\alpha-m}\left\{\exp \left(-\alpha t_{0}+m t_{0}\right)\right. \\
& \left.-\exp (-\alpha t+m t)\})^{2}\right] d t_{0},
\end{aligned}
$$

respectively.

\subsubsection{Spatially and temporally dependent velocity and temporally dependent decay term}

Let us consider $\phi(t)=1$, so from equation (7), $u(x, t)=u_{0}+\alpha x g(t)$, where a form, $g(t)=$ $1 /\left(1+m_{1} t\right)$ is considered and $m_{1}$ is another temporal dependence parameter of the same dimension of $m$, but different values are considered for the both. From equations (13 and 21), we have $\beta(t)=$ $\left(1+m_{1} t\right)^{\alpha / m_{1}}$ and $T=\int_{0}^{t} \frac{v}{v+k}\left(1+m_{1} v\right)^{-2 \alpha / m_{1}} d v$, respectively. Using equation (30), we obtain the solution for instantaneous injection as:

$$
\begin{aligned}
c(x, t)= & M \frac{\left(1+m_{1} t\right)^{-\alpha / m_{1}}}{\sqrt{4 \pi D_{0} T}} \\
& \times \exp \left[-\frac{1}{4 D_{0} T}\left(\frac{x}{\left(1+m_{1} t\right)^{\alpha / m_{1}}}\right.\right. \\
& \left.\left.-\frac{u_{0}}{m_{1}-\alpha}\left\{\left(1+m_{1} t\right)^{1-\alpha / m_{1}}-1\right\}\right)^{2}\right],
\end{aligned}
$$

and that for a continuous injection, may be obtained from solution (32) as:

$$
\begin{aligned}
c(x, t)=C_{0} & u_{0} \int_{0}^{t} \frac{\left(1+m_{1} t\right)^{-\alpha / m_{1}}}{\sqrt{4 \pi D_{0}(T-\zeta)}} \\
& \times \exp \left[-\frac{1}{4 D_{0}(T-\zeta)}\left(\frac{x}{\left(1+m_{1} t\right)^{\alpha / m_{1}}}\right.\right. \\
& -\frac{u_{0}}{m_{1}-\alpha}\left\{\left(1+m_{1} t\right)^{1-\alpha / m_{1}}\right. \\
& \left.\left.\left.-\left(1+m_{1} t_{0}\right)^{1-\alpha / m_{1}}\right\}\right)^{2}\right] d t_{0}
\end{aligned}
$$




\section{Discussion}

To discuss the different solutions obtained as in the previous section, they are illustrated in the domain $0 \leq x$ (meter) $\leq 100$ at different time. The input values are chosen same as considered in the paper (Basha and El-Habel 1993) to compare the results and figures of both the works. These are: injected mass, $M=1.0$, dispersion coefficient and velocity in homogeneous medium $D_{0}\left(\mathrm{~m}^{2} / \mathrm{hr}\right)=$ 1.0 , and $u_{0}(\mathrm{~m} / \mathrm{hr})=0.25$, respectively. Figures 1 and 2 illustrate the solution (33) for instantaneous point source which is discussed in subsection 2.3.1. Figure 1 is drawn at $t(\mathrm{hr})=5,50$, and 200 for two

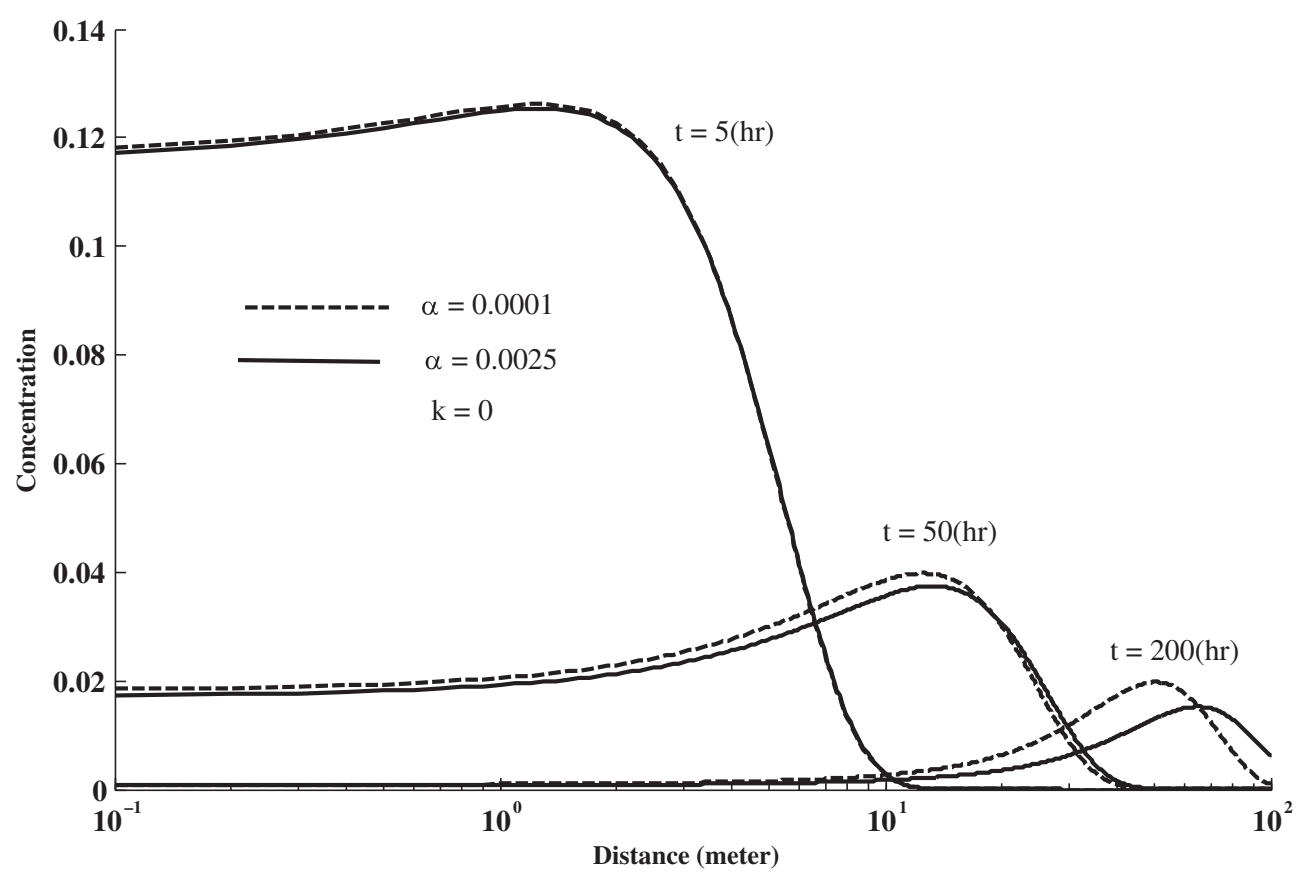

Figure 1. Concentration distribution from instantaneous injection source along homogeneous medium (dotted curves) and heterogeneous medium (solid curves), represented by equation (33) for constant dispersion coefficient.

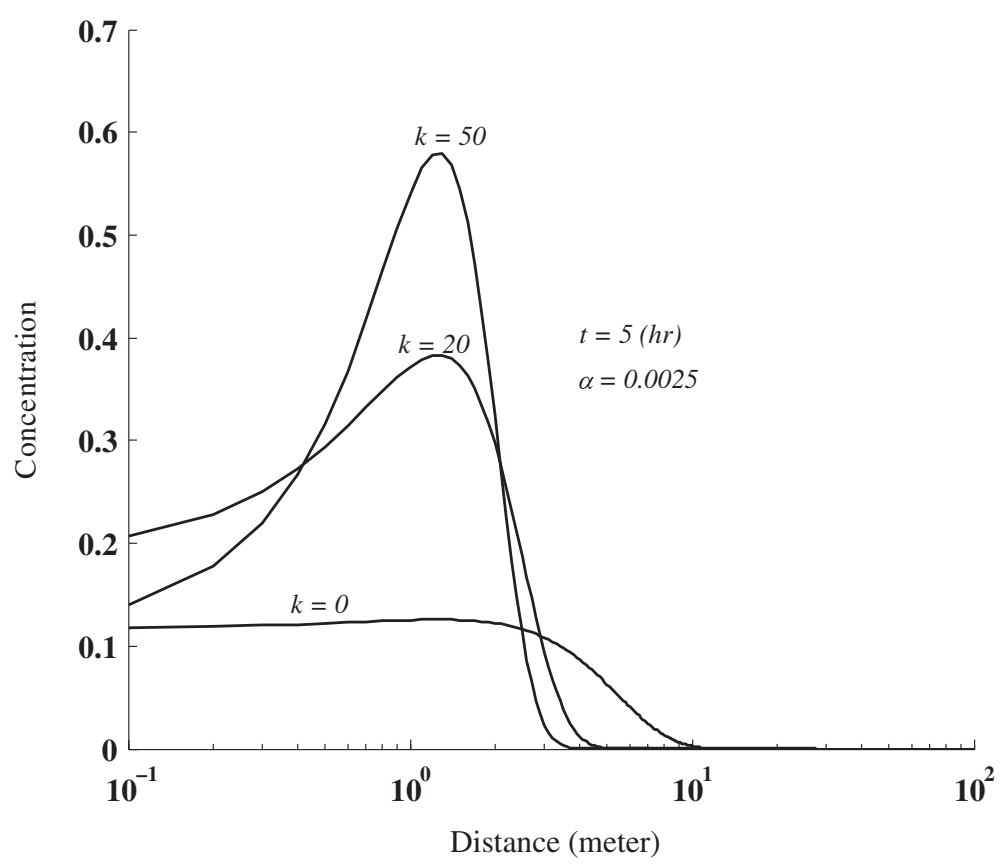

Figure 2. Comparison of concentration levels from instantaneous source due to constant dispersion and asymptotic dispersion along heterogeneous medium represented by equation (33). 
values of spatial dependence parameter $\alpha=0.0025$ and $\alpha(\mathrm{hr})^{-1}=0.0001$. The dispersion coefficient is considered constant (first in the four forms discussed in this subsection). Solid curves and dotted curves are drawn for the higher and lower values of $\alpha$, respectively. As the former value of $\alpha$ is much higher as compared to its latter value so it represents the velocity varying linearly with position and the latter value represents the uniform velocity, i.e., $u=u_{0}$ (see section 2.3.1). The dotted curves are exactly the same curves at $k=0$ of figure 2 of the paper (Basha and El-Habel 1993). It may be noted that the solution in equation (23) of Basha and El-Habel (1993) for which they have drawn figure 2 may be obtained from our solution given by equation (33) under the limit $\alpha \rightarrow 0$. The resemblance of solid curves with the dotted curves validates the correctness of the solution in equation (33) of the present work. The figure reveals that increasing value of velocity causes faster solute transport characterized by lower concentration peak as compared to the homogeneous medium (represented by uniform velocity and dispersion). Figure 1 reveals the concentration distribution pattern originating from an instantaneous source. The concentration peak is the highest and proximal to the injected location at $t=5(\mathrm{hr})$.
It attenuates and drifts forward with time and position.

In rest of the figures, $\alpha(\mathrm{hr})^{-1}=0.0025$ is taken, which implies heterogeneity of the medium. Figure 2 exhibits the same solution in equation (33) of section 2.3.1, but for the fourth form of the dispersion coefficient which is asymptotic. This figure compares the concentration pattern represented by the solution in equation (33) for constant dispersion coefficient $(k=0)$, with those for asymptotic dispersion coefficient for $k(\mathrm{hr})=20$ and 50 at $t(\mathrm{hr})=5$. This figure reveals the expected pattern of the peak concentration increasing significantly and approaching towards the source location, with $k$. Figure 3 illustrates the solutions given by equations (39), (41) and (43) discussed in section 2.3.2 for three expressions of $\phi(t)$, and for asymptotic dispersion coefficient using $k=20$ at $t(\mathrm{hr})=5$. The pattern of concentration dispersion in each case is very much congruous with the expressions of $\phi(t)$. The value of temporal dependence parameter $m(\mathrm{hr})^{-1}$ is taken as 0.1. The curve for $\phi(t)=$ 1 in figure 3 may also be visualized in figure 2 , the case for which the solution is given in equation (33). Figure 4 depicts the concentration values evaluated from the solution in equation (45) discussed in section 2.3.3 at $t(\mathrm{hr})=5,50$, and 200. The dispersion

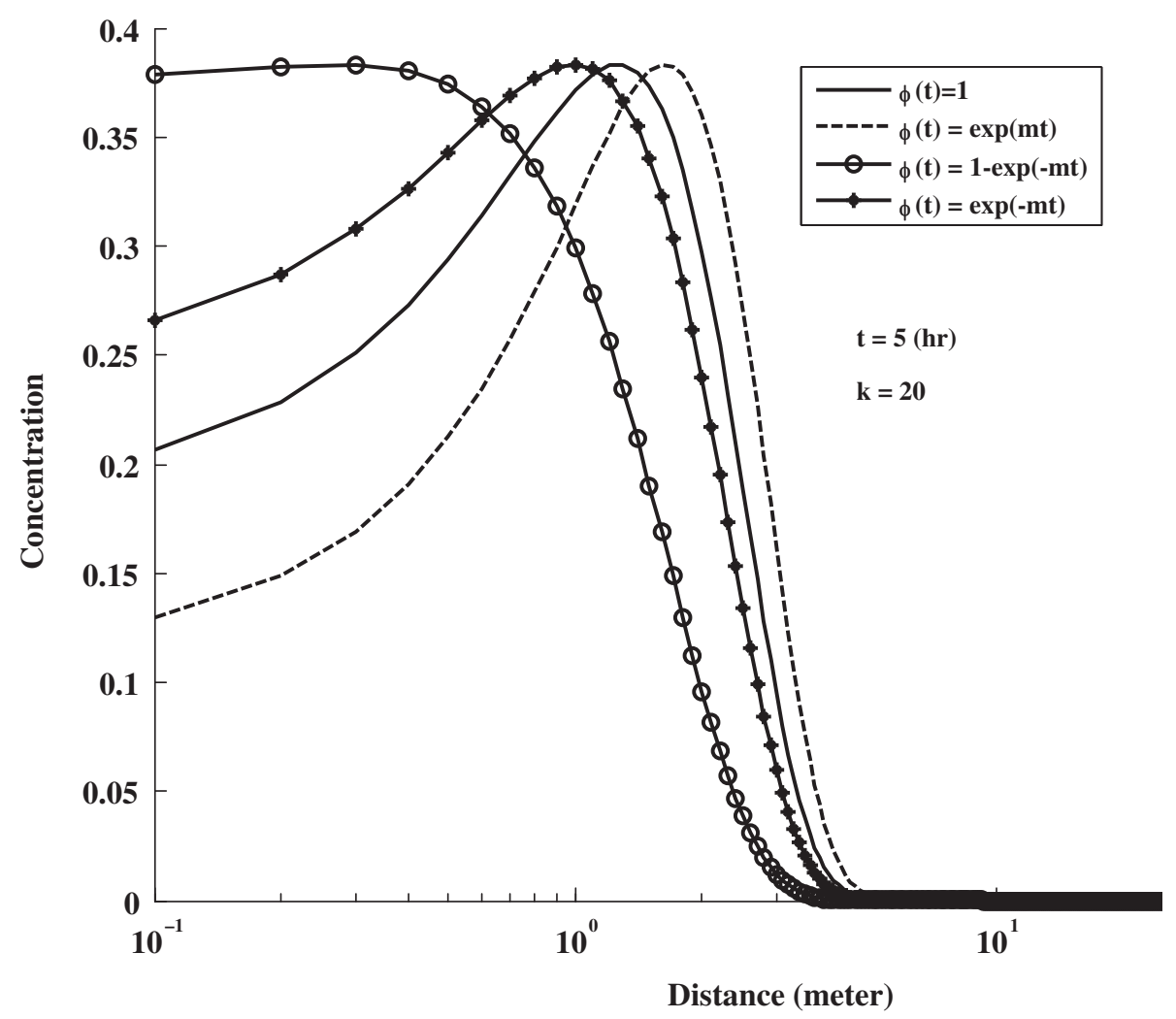

Figure 3. Comparison of concentration levels from instantaneous source due to asymptotic dispersion coefficient represented by equations $(33,39,41$ and 43$)$. 


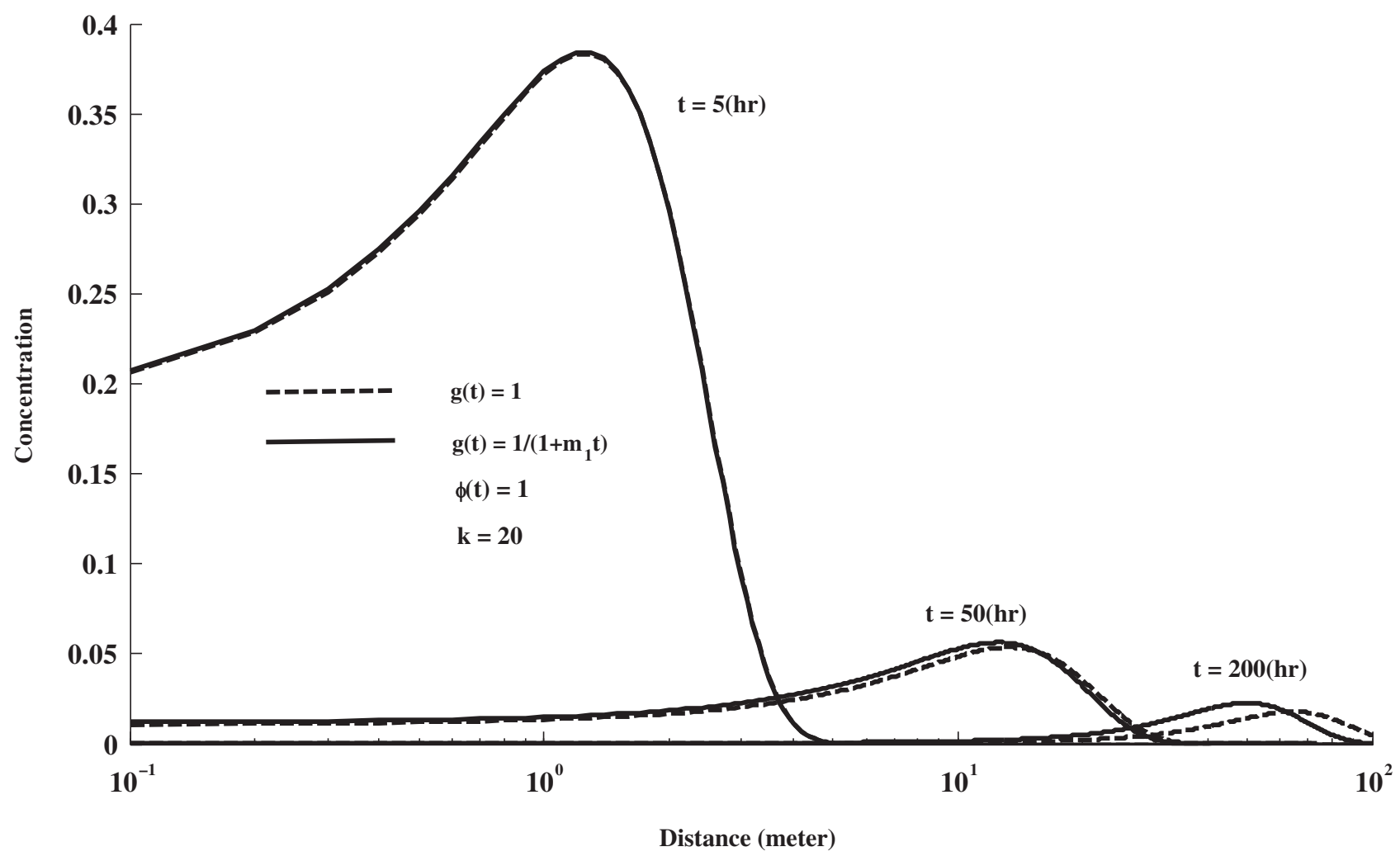

Figure 4. Comparison of concentration levels from instantaneous source due to asymptotic dispersion coefficient represented by equations (33 and 45$)$.

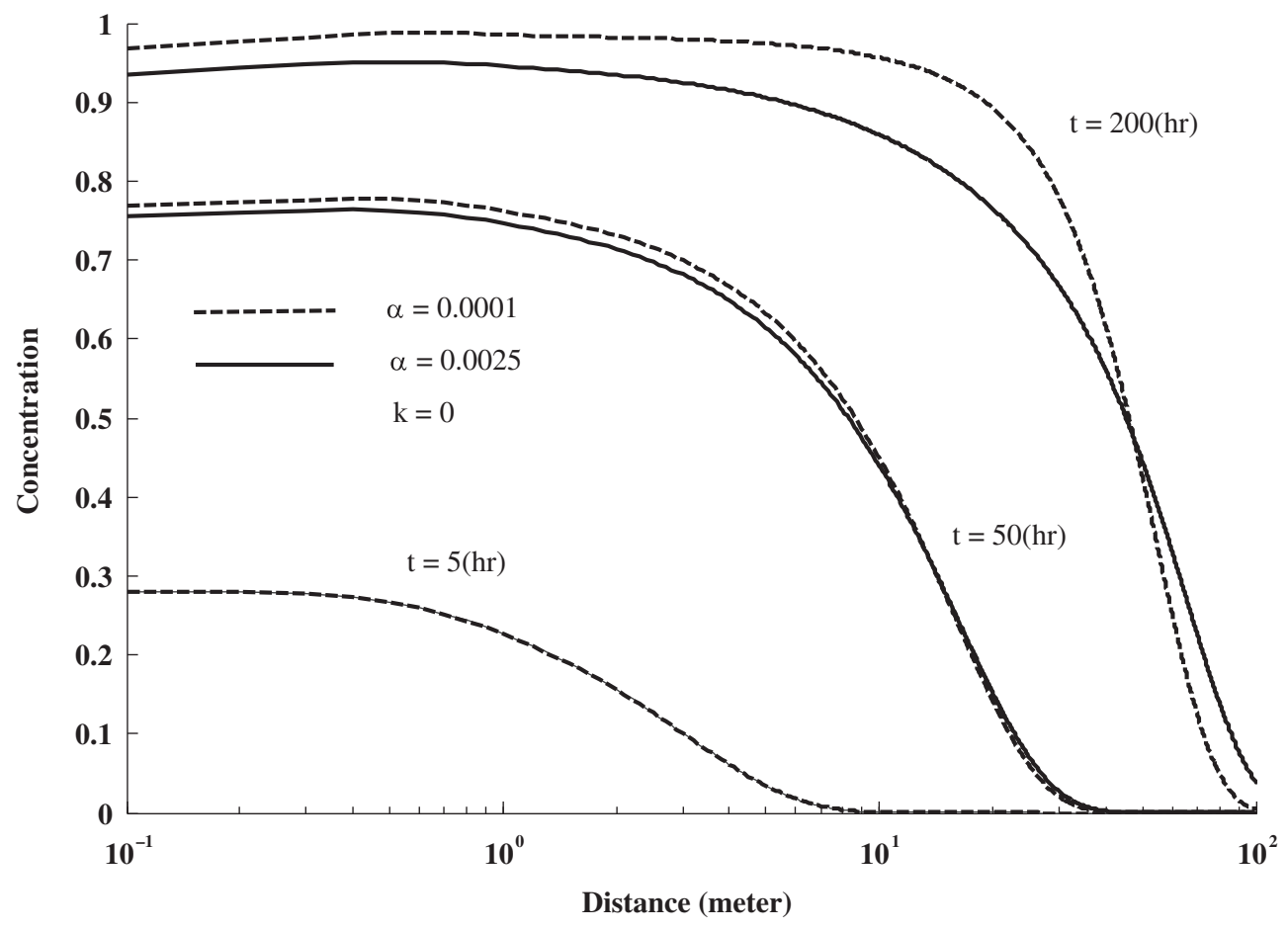

Figure 5. Concentration distribution from continuous source along homogeneous medium (dotted curves) and heterogeneous medium (solid curves) represented by equation (34).

coefficient is again considered asymptotic using $k=20$. The temporal dependent decay term occurring in the expression of the velocity is assumed as $g(t)=1 /\left(1+m_{1} t\right)$, i.e, velocity decreases with time hence, same is the pattern of the concentration level. Another temporal dependence parameter is 
chosen as $m_{1}(\mathrm{hr})^{-1}=1.0$ to draw this figure. Figure 5 is drawn for solution (34) obtained for continuous source discussed in section 2.3.1 for the constant dispersion coefficient, at $t(\mathrm{hr})=5,50$ and 200 for the same two values of $\alpha$. The solid curves are drawn for $\alpha=0.0025$ showing the effect of increasing velocity with position on the concentration pattern. The dotted curves $(\alpha=0.0001)$ match with the curves of figure 3 for $k=0$ (Basha and El-Habel 1993).

\section{Conclusions}

In a previous paper (Basha and El-Habel 1993), the heterogeneity of the medium has been described by time-dependent dispersion coefficient of the ADE and velocity has been considered uniform. The present work extends this work by considering velocity as spatially and temporally dependent and dispersion coefficient as temporally dependent. This assumption is based on the conclusions of the paper (Sternberg et al. 1996). It describes the heterogeneity of the medium in more general way than in previous works. A moving coordinate transformation is introduced to reduce the ADE of the present formulation into the one considered in the paper (Basha and El-Habel 1993). The results of both the works are matched through particular solutions and figures for instantaneous and continuous sources. The resemblance between the results and figures of both the works validates our formulation and the solutions obtained through the transformation derived. Analytical solutions of $\mathrm{ADE}$ with variety of expressions in terms of position and time variables for its both the coefficients with the help of appropriately derived transformation equations will be the main task of the author's future works.

\section{Acknowledgements}

The first author expresses his gratitude to University Grants Commission, Government of India, for financial and academic assistance in the form of Senior Research Fellowship. The authors express their gratitude to the reviewers for their valuable comments and suggestions which improved the present work to a great extent.

\section{List of symbols}

$c$ : $\quad$ Solute concentration in domain $x$

$C$ : Solute concentration in new domain $X$

$C_{i}$ : Initial concentration

$C_{0}$ : Reference concentration

$D_{0}$ : Dispersion coefficient in homogeneous medium $u_{0}: \quad$ Velocity in homogeneous medium

$K$ : $\quad$ Solute concentration in the new domain $\eta$

$M: \quad$ Injected pollutant mass

$k$ : $\quad$ Asymptotically varying parameter

$\nu, t_{0}$

$\chi, \varsigma, \xi:$ Dummy variables

$m: \quad$ Temporal dependence parameter

$m_{1}$ : Another temporal dependence parameter

$x$ : Position variable

T: $\quad$ Another new time variable

$t$ : $\quad$ Time variable

$t^{\prime}$ : $\quad$ Time in new domain

$X: \quad$ Position in new domain

$\eta: \quad$ A new space variable

$\alpha: \quad$ Coefficient in the decay term

$\delta(\cdot)$ Dirac delta function

\section{References}

Anderson M 1979 Modeling of groundwater flow systems as they relate to the movement of contaminants; CRC Crit. Rev. Environ. Control 9 97-156.

Aral M M and Liao B 1996 Analytical solutions for twodimensional transport equations with time-dependent dispersion coefficients; J. Hydrol. Eng. 1 20-32.

Basha H A and El-Habel F S 1993 Analytical solution of the one-dimensional time dependent transport equation; Water Resour. Res. 29(9) 3209-3214.

Beck J V, Cole K D and Litkouhi B 1992 Heat conduction using Green's function; Hemisphere Publishing Co., Washington, D.C.

Crank J 1975 Mathematics of Diffusion; Oxford University Press, New York.

Dagan G 1987 Theory of solute transport by groundwater; Ann. Rev. Fluid Mech. 19 183-215.

Gelhar L W, Mantoglou A, Welty C and Rehfeldt K R 1985 A review of field-scale physical transport processes in saturated and unsaturated porous media; EPRI Rep. EA-4190, Elec. Power Res. Inst., Palo Alto, Calif.

Güven O, Molz F J and Melville J G 1984 An analysis of dispersion in a stratified aquifer; Water Resour. Res. 20(10) 1337-1354.

Haberman R 1987 Elementary applied partial differential Equations; Prentice-Hall, Englewood Cliffs, New Jersey.

Kumar A, Jaiswal D K and Kumar N 2009 Analytical solutions of one-dimensional advection-diffusion equation with variable coefficients in a finite domain; J. Earth Syst. Sci. 118(5) 539-549.

Leij F J and Van Genuchten M 2000 Analytical modeling of nonaqueous phase liquid dissolution with Green's functions; Transp. Porous Media 38 141-166.

Matheron G and de Marsily G 1980 Is transport in porous media always diffusive? A counter example; Water Resour. Res. 16 901-917.

Park E and Zhan H 2001 Analytical solutions of contaminant transport from finite one-, two-, and three-dimensional sources in a finite-thickness aquifer; J. Contam. Hydrol. 53 41-61.

Pickens J F and Grisak G E 1981a Scale-dependent dispersion in stratified granular aquifer; Water Resour. Res. 17(4) 1191-1211. 
Pickens J F and Grisak G E 1981b Modeling of scaledependent dispersion in hydrogeologic systems; Water Resour. Res. 17(6) 1701-1711.

Simpson E S 1978 A note on the structure of the dispersion coefficient; Geol. Soc. Am. Abstr. Programs, 393p.

Singh M K, Mahato N K and Singh P 2008 Longitudinal dispersion with time-dependent source concentration in semi-infinite aquifer; J. Earth Syst. Sci. 117(6) 945-949.

Sposito G W, Jury W A and Gupta V K 1986 Fundamental problems in the stochastic convection-dispersion model of solute transport in aquifers and field soils; Water Resour. Res. 22 77-78.

Sternberg S P K, Cushman J H and Greenkorn R A 1996 Laboratory observation of nonlocal dispersion; Trans. Porous Media 13 123-151.

Su N, Sander G C, Liu F, Anh V and Barry D A 2005 Similarity solutions for solute transport in fractal porous media using a time- and scale-dependent dispersivity; Appl. Math. Model. 29(9) 852-870.

Van Genuchten M Th, Leij F J, Skaggs T H, Toride N, Bradford S A and Pontedeiro E M 2013 Exact analytical solutions for contaminant transport in rivers. 1 . The equilibrium advection-dispersion equation; J. Hydrol. Hydromech. 61(2) 146-160.

Wang S T, McMillan A F and Chen B H 1977 Analytical model of dispersion in tidal fjords; J. Hydraul. Div. ASCE 103(HY7) 737-751.

Yadav S, Kumar A, Jaiswal D K and Kumar N 2011 Onedimensional unsteady solute transport along unsteady flow through inhomogeneous medium; J. Earth Syst. Sci. 120(2) 205-213.

Yeh G T 1981 AT123D: Analytical transient one-, two-, and three-dimensional simulation of waste transport in the aquifer system. Envir. Sci. Div. 1439, Report ORNL5602, Oak Ridge, Tennessee, USAp.

Yeh G T and Yeh H D 2007 Analysis of point-source and boundary-source solutions of one-dimensional groundwater transport equation; J. Environ. Eng. 133(11) 1032-1041.

Zamani K and Bombardelli F A 2012 One-dimensional, mass conservative, spatially-dependent transport equation: New analytical solution; 12th Pan-American Congress of Applied Mechanics, 2-6 January, 2012, Port of Spain, Trinidad.

MS received 4 December 2015; revised 27 August 2016; accepted 2 September 2016

Corresponding editor: Subimal GHosh 